\title{
Darier's Disease Flare following COVID-19 Vaccine
}

\author{
Mette Vestergaard Elbæk ${ }^{a} \quad$ Gabrielle Randskov Vinding ${ }^{a}$ \\ Gregor Borut Ernst Jemec ${ }^{a, b}$ \\ aDepartment of Dermatology, Zealand University Hospital, Roskilde, Denmark; 'b Institute \\ of Clinical Medicine, Faculty of Health and Medical Sciences, University of Copenhagen, \\ Copenhagen, Denmark
}

\section{Keywords}

Darier's disease $\cdot$ Disease flare $\cdot$ Genodermatosis $\cdot$ COVID-19 vaccine $\cdot$ Vaccination

\begin{abstract}
Darier's disease is a rare genodermatosis characterized clinically by dyskeratotic papules in the seborrheic and intertriginous areas and nail abnormalities. Dyskeratosis and acantholysis are typical histological findings. Darier's disease is not known to be inflammatory by nature as inflammation occurs primarily due to local infections, and it may therefore differ from inflammatory dermatoses such as psoriasis and cutaneous lupus in response to antigen stimulation. Known triggers of Darier's disease primarily include exogenous factors such as sun exposure, friction, or infection. We present a case of a 47-year-old white female with a flare of Darier's disease 2 days following her first vaccination with COVID-19 vaccine (ChAdOx1-s [recombinant]) (Vaxzevria ${ }^{\circledR}$ [previously known as COVID-19 vaccine AstraZeneca]). In this case report, we discuss possible mechanisms linking the vaccination and the flare of Darier's disease. We consider inflammatory mechanisms as well as a random co-occurrence. Due to the close time-related association between the disease flare and the COVID-19 vaccination, we find an urge to make other clinicians aware of a possible association.
\end{abstract}

(c) 2021 The Author(s).

Published by S. Karger AG, Basel

\section{Introduction}

Darier's disease is a rare autosomal dominantly inherited dermatosis characterized clinically by dyskeratotic papules in the seborrheic and intertriginous areas and nail abnormalities. The genetic abnormality is due to a mutation in the ATP2A2 gene on chromosome

Gabrielle Randskov Vinding and Gregor Borut Ernst Jemec share senior authorship for this study. 
12q23-24 which encodes SERCA2, a calcium ATPase in the sarco/endoplasmic reticulum. Dyskeratosis and acantholysis are typical histological findings [1]. Inflammation occurs primarily due to local infections.

In contrast to, for example, psoriasis, Darier's disease is thus not known to be inflammatory by nature and may therefore differ from inflammatory dermatoses such as psoriasis and cutaneous lupus in response to antigen stimulation. The inflammatory dermatoses are often inherently dynamic and may flare after any antigen stimulation. An antigen-associated flare of Darier's disease therefore suggests involvement of additional mechanisms. We present a case of a patient with a flare of Darier's disease following her first vaccination with COVID-19 vaccine (ChAdOx1-s [recombinant]) (Vaxzevria ${ }^{\circledR}$ [previously known as COVID-19 vaccine AstraZeneca]) and discuss possible mechanisms linking the vaccination and the flare of Darier's disease.

\section{Case Report}

The patient was a 47-year-old white female with histologically verified Darier's disease since the age of 18 . She had attended regular follow-up visits at a department of dermatology for 10 years at which point the patient had clinical remission, and further follow-up was deemed futile. Previous treatments had included topical and systemic retinoids, topical steroids, and disinfectants. The patient had stopped the regular consultations at the department 4 months prior to her COVID-19 vaccination. Two days following vaccination, the patient experienced flu-like symptoms including fever and a rash affecting intertriginous areas, chest, upper abdominal, and lower back areas. Initially, the patient did not link the rash to her Darier's disease as she had not previously experienced the disease this severely. She was re-referred to the Department of Dermatology, and at clinical examination 3 weeks after vaccination, the patient presented as shown in Figure 1 with dyskeratotic papules in the abovementioned regions in a pattern of findings typical for Darier's disease. Due to previous poor response to local retinoids, we initiated treatment with a combination of topical corticosteroids and salicylic acid in an effort to alleviate the symptoms of inflammation. Due to minimal effect at consultation, 3 weeks later oral isotretinoin was prescribed targeting core pathological processes with good clinical response. The patient will not get a second dose of this vaccine as The Danish Medical Agency has suspended the use of Vaxzevria ${ }^{\circledR}$.

\section{Discussion}

We report a flare of Darier's disease following COVID-19 vaccination. Well-known triggers of Darier's disease primarily include exogenous factors such as sun exposure, friction, or infection [2]. In addition, case reports describe flares of Darier's disease following treatment of interferon therapy $[3,4]$ and lithium $[5,6]$ indicating involvement of a possible immune mechanism.

The possible mechanism between flare of Darier's disease following COVID-19 vaccination is however unclear. Generally, the causes of adverse events following immunization as described by the WHO [7] may be related to the vaccine (product-related or quality defectrelated), the patient (immunization anxiety-related or coincidental event), or immunization error-related (handling, prescribing, or administrating the vaccine).

Well-known side effects of the vaccine include local reactions at the injection site, fatigue, headache, myalgia, and fever [8]. Dermatological side effects to vaccines against COVID-19 have been described in 2 case reports ( 3 patients) $[9,10]$, but no studies to date report flares

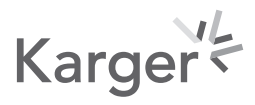


Fig. 1. Photo of the patient in the case presenting with flare of Darier's disease.

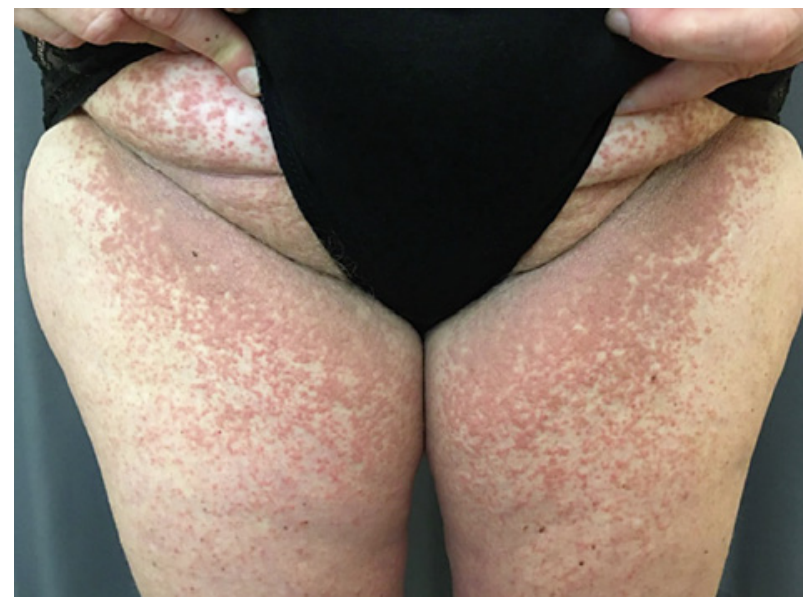

of existing skin disease following COVID-19 vaccination. One study described no flares in a cohort of 26 patients with chronic inflammatory disease (among these, 4 patients with psoriasis and 1 patient with systemic lupus erythematosus) compared to healthy controls after receiving anti-SARS-CoV-2 mRNA vaccines [11]. However, flare of an inflammatory skin disease after other vaccinations is well described, for example, after influenza vaccination in psoriasis [12-14] and after hepatitis B vaccination in systemic lupus erythematosus [15] and in lupus panniculitis [16].

Adverse vaccine events may involve immune-mediated phenomena triggered by exposure to the microbial or other components of vaccines [17]. Cytokine response following the vaccine could potentially result in a flare of Darier's disease since proinflammatory cytokines IL-6 and IL-8 have been investigated to play important roles in the regulation of expression of ATP2A2 and ATP2C1 in cultured normal human keratinocytes [18]. However, in a clinical trial, the cytokine levels found to be significantly increased in individuals who received COVID-19 vaccine (ChAdOx1-s [recombinant]) were interferon-gamma and IL-2 [19].

We cannot rule out a pure random co-occurrence of our patient receiving the COVID-19 vaccine and a following flare of Darier's disease without causation, but we find the close timerelated association between the vaccine and the disease flare striking and find an urge to make other clinicians aware of a possible association. An unbiased study, that is, reporting both flares and absence of flares in consecutive patients, may help the identification of the underlying mechanism.

\section{Statement of Ethics}

The research was conducted ethically in accordance with the World Medical Association Declaration of Helsinki. Written informed consent was obtained from the patient for publication of this case report and the accompanying image.

\section{Conflict of Interest Statement}

M.V. Elbæk has no conflicts of interest to declare. G.R. Vinding has no conflicts of interest to declare. G.B.E. Jemec has received honoraria from AbbVie, Chemocentryx, Coloplast, Incyte, Inflarx, Kymera, Leo Pharma, Novartis, UCB, and Wella for participation on advisory boards, 
grants from AbbVie, AstraZeneca, CSL, Inflarx, Janssen-Cilag, Leo Pharma, Novartis, Regeneron, and Sanofi for participation as an investigator, and received speaker honoraria from AbbVie, Boehringer-Ingelheim, Galderma, and Novartis. He has also received unrestricted departmental grants from Leo Pharma and Novartis.

\section{Funding Sources}

No funding was received.

\section{Author Contributions}

All 3 authors have contributed to the idea of the case report and design of the manuscript. M.V.E. has drafted the manuscript and taken the clinical photograph for Figure 1. G.R.V. and G.E.B.J. have revised the manuscript critically. All 3 authors have approved of the final version of the manuscript.

\section{Data Availability Statement}

No data are associated with this article.

\section{References}

1 Cooper SM, Burge SM. Darier's disease: epidemiology, pathophysiology, and management. Am J Clin Dermatol. 2003;4(2):97-105.

2 Burge SM, Wilkinson JD. Darier-White disease: a review of the clinical features in 163 patients. J Am Acad Dermatol. 1992 Jul;27(1):40-50.

3 Castagna J, Clerc C, Dupond AS. Exacerbation of Darier disease by peg-interferon beta-1a in a patient suffering from multiple sclerosis. Int J Dermatol. 2018;57(1):116-7.

4 Karagiannidis I, Brunner M, Zouboulis CC. Exacerbation of darier disease under interferon- $\alpha$-2a therapy with clinical signs of lichen nitidus. Case Rep Dermatol. 2016;8(2):218-23.

5 Ngo J, Haber R. Exacerbation of Darier disease by lithium carbonate. J Cutan Med Surg. 2010;14(2):80-4.

6 Milton GP, Peck GL, Fu JJ, DiGiovanna JJ, Nordlund JJ, Thomas JH, et al. Exacerbation of Darier's disease by lithium carbonate. J Am Acad Dermatol. 1990 Nov;23(5 Pt 1):926-8.

7 CIOMs. Vaccine safet y BaSicS. World Heal Organ [Internet]. 2013. Available from: http://vaccine-safetytraining.org/overview-and-outcomes-1.

8 Kaur RJ, Dutta S, Bhardwaj P, Charan J, Dhingra S, Mitra P, et al. Adverse events reported from COVID-19 vaccine trials: a systematic review. Indian J Clin Biochem. 2021 Mar;1-13.

9 Lam M, Egail M, Bedlow AJ, Tso S. Ribonucleic acid (RNA) COVID-19 vaccine associated cutaneous adverse drug events: a case series of two patients. Clin Exp Dermatol. 2021.

10 Jedlowski PM, Jedlowski MF. Morbilliform rash after administration of Pfizer-BioNTech COVID-19 mRNA vaccine. Dermatol Online J. 2021 Jan;27(1).

11 Geisen UM, Berner DK, Tran F, Sümbül M, Vullriede L, Ciripoi M, et al. Immunogenicity and safety of anti-SARSCoV-2 mRNA vaccines in patients with chronic inflammatory conditions and immunosuppressive therapy in a monocentric cohort. Ann Rheum Dis. 2021 Mar.

12 Munguía-Calzada P, Drake-Monfort M, Armesto S, Reguero-Del Cura L, López-Sundh AE, González-López MA. Psoriasis flare after influenza vaccination in Covid-19 era: a report of four cases from a single center. Dermatol Ther. 2021;34:e14684.

13 Gunes AT, Fetil E, Akarsu S, Ozbagcivan O, Babayeva L. Possible triggering effect of influenza vaccination on psoriasis. J Immunol Res. 2015;2015:258430.

14 Sbidian E, Eftekahri P, Viguier M, Laroche L, Chosidow O, Gosselin P, et al. National survey of psoriasis flares after 2009 monovalent H1N1/seasonal vaccines. Dermatology. 2014;229(2):130-5.

15 Agmon-Levin N, Zafrir Y, Paz Z, Shilton T, Zandman-Goddard G, Shoenfeld Y. Ten cases of systemic lupus erythematosus related to hepatitis B vaccine. Lupus. 2009 Nov;18(13):1192-7. 
16 Choffray A, Pinquier L, Bachelez H. Exacerbation of lupus panniculitis following anti-hepatitis-B vaccination. Dermatology. 2007;215(2):152-4.

17 Siegrist C-A. Mechanisms underlying adverse reactions to vaccines. J Comp Pathol. 2007 Jul;137 Suppl l:S4650.

18 Mayuzumi N, Ikeda S, Kawada H, Ogawa H. Effects of drugs and anticytokine antibodies on expression of ATP2A2 and ATP2C1 in cultured normal human keratinocytes. Br J Dermatol. 2005 May;152(5):920-4.

19 Ewer KJ, Barrett JR, Belij-Rammerstorfer S, Sharpe H, Makinson R, Morter R, et al. T cell and antibody responses induced by a single dose of ChAdOx1 nCoV-19 (AZD1222) vaccine in a phase 1/2 clinical trial. Nat Med. 2021 Feb;27(2):270-8. 